\title{
UTILITY OF SEROLOGY IN THE DIAGNOSIS OF AMOEBIC HEPATITIS AND HYDATID DISEASE AMONG PATIENTS WITH BENIGN HEPATIC CYSTS.
}

\author{
By \\ Hosam El-Nemr*, Khaled Farid ${ }^{\star *}$, Saher Taman ${ }^{\star \star *}$, \\ Elham Farag Almeniar*, Amira Taman*

\begin{abstract}
Trom
Department of Medical Parasitology * Tropical Medicine **,

Radiology ${ }^{* * *}$ Faculty of Medicine, Mansoura University
\end{abstract}

\begin{abstract}
Background: Parasites as hydatid disease and amoebic liver abscess can cause cystic lesions in the liver and their diagnosis depends on detection of IgG antibodies in the serum in combination with imaging techniques.
\end{abstract}

Aim of the work: To evaluate both sensitivity and specificity of ELISA test as a serological method for detection of IgG antibodies in patients with hydatid disease and amoebic liver abscess among patients with cystic lesions of the liver.

Methodology: A case-control study was conducted on 48 patients with cystic lesions in the liver suspi- cious to be hydatid cyst or amoebic liver abscess, during the period from October 2012 to December 2013. Patients involved in the study were admitted to Mansoura University Hospital at the Tropical Medicine Department and were tested for antihydatid and anti-amoebic IgG antibodies using ELISA technique.

Results: Thirty-one suspicious cases of hydatid disease were examined by histopathology, as a gold standard test, later; they were tested for IgG antibodies using ELISA. Histopathology detected eleven cases as hydatid disease in the liver, however, ELISA test recorded 10 positive cases and one case gave a false negative reaction, with sensitivity

MANSOURA MEDICAL JOURNAL 
and specificity $90.9 \%$ and $95 \%$ respectively. Seventeen suspicious cases of hepatic abscess were tested using IHA for anti-amoebic IgG antibodies, only 2 positive cases of amoebic liver abscess were confirmed, the two cases were also positive when using the ELISA test with sensitivity and specificity $100 \%$ and 93.3\% respectively.

Conclusion: ELISA is a highly sensitive and specific serological technique for diagnosis of hydatid disease and amoebic liver abscess among patients with cystic lesions of the liver.

\section{INTRODUCSTION}

Cystic lesions of the liver include wide spectrum of disorders with increased incidence nowadays due to the frequent use of abdominal imaging techniques (1). Hydatid disease and Amoebic Liver Abscess are known parasitic causes of hepatic cystic lesions (2).

Hydatid disease is a neglected zoonotic disease caused by Echinococcus granulosus, with worldwide distribution, but highest prevalence occur in Mediterranean countries including Egypt (3). As most hydatid cysts remain clinically silent and the clinical examination is unreliable, diagnosis depends on imaging techniques and serological tests, mainly antibody detection (4).

Amoebic liver abscess (ALA) is caused by protozoan parasite Entamoeba histolytica, a common parasitic infection in tropical countries (5). Detection of trophozoites using microscopic examination of liver aspirate, though confirmatory of ALA, is quite insensitive (6). Diagnosis of ALA is frequently conducted using serum anti-amoebic $\lg G$ antibodies (7).

The aim of this work was to evaluate the sensitivity and specificity of ELISA test for detection of hydatid disease in the liver and ALA among patients with hepatic cystic lesions.

\section{SUBJECTS, MATERIALS AND METHODS}

This study was conducted, during the period from October 2012 to December 2013, on 48 patients with benign hepatic cysts; suspected to be parasitic in origin who were admitted to Mansoura University Hospital at the Tropical Medicine Department. Patients were subjected 
to complete history taking, clinical examination and abdominal ultrasonography to evaluate hepatic cysts which could be hydatid disease or amoebic liver abscess. A blood sample $(5 \mathrm{ml})$ was taken from all patients, left to clot and serum was obtained by centrifugation then stored at $-20^{\circ} \mathrm{C}$ until use for detection of IgG antibodies against $E$. granulosus and E. histolytica using Echinococcus ELISA (Cat\# 8202-35, Diagnostic Automation, USA) and Entamoeba histolytica Amebiasis ELISA (Cat\# 8201-35, Diagnostic Automation, USA). Briefly, serum samples were diluted using dilution buffer supplied with the kit and incubated with the coated plates for 10 min at room temperature. After washing, an enzyme conjugate was added for $5 \mathrm{~min}$, followed by washing and finally, chromogen was added and the reaction was stopped using stop buffer. Negative and positive controls included in the kit were tested to check validity of the kit. In addition, healthy controls, 11 persons, were tested to check for false results. Moreover, 7 patients with other parasitic infections (3 schistosomiasis mansoni, 2 toxoplasmosis and 2 giardiasis) were used to test for cross reactivity.
Ethical consideration

This study was approved by medical ethics committee of Faculty of Medicine, Mansoura University, Egypt. Informed and written consents were obtained from all participants in the study.

\section{Statistical analysis}

Data were statistically analyzed using EXCEL to obtain analytical statistics [sensitivity, specificity, diagnostic accuracy, positive predictive values (PPV) and negative predictive values (NPV)] of each assay.

\section{RESULTS}

Thirty-one cases of clinically and radiologically suspicious hydatid disease in the liver were examined by ultrasonography, CT (Fig. 1), IHA in addition to histopathology. Eleven cases were proved to be positive for hydatid disease by histopathology (Fig. 2) and 20 cases were free of the disease. All the 31 case were tested for anti-hydatid IgG antibodies by ELISA technique. From the 11 confirmed cases, only one case gave false negative by ELISA test (Table 1). Sensitivity and specificity of ELISA test for diagnosis of hydatid liver disease were calculated as shown in (Table 2). Serum samples

MANSOURA MEDICAL JOURNAL 
4 UTILITY OF SEROLOGY IN THE DIAGNOSIS etc...

from controls used in the study were tested using ELISA test and only one sample from schistosomiasis patient had cross-reactivity with the kit (Table 3). Seventeen suspicious cases of hepatic abscess were tested for liver amoebic infection using IHA and ELISA kit and in both, two cases only were recorded as positive for amoebic liver abscess using
IHA and ELISA, however suspected case recorded negative by IHA but was positive (false positive) with ELISA test (Table 4). The sensitivity and specificity of ELISA test were $100 \%$ and $93.3 \%$ respectively (Table 5). From the controls, only one case of Giardia showed crossreactivity with the tested ELISA kit (Table 6).

Table 1. Suspected cases of hydatid disease and their reaction with ELISA test.

\begin{tabular}{|l|l|l|}
\hline & Patients with disease & Patients without disease \\
\hline ELISA test positive & 10 & 1 \\
\hline ELISA test negative & 1 & 19 \\
\hline
\end{tabular}

Table 2. Sensitivity and specificity of ELISA test for hydatid disease patients

\begin{tabular}{|l|l|l|l|l|l|}
\hline Test & Sensitivity & Specificity & PPV & NPV & Diagnostic Efficiency \\
\hline ELISA & $90.9 \%$ & $95 \%$ & 90.9 & 95 & 93.5 \\
\hline
\end{tabular}


Table 3. Detection of antihydatid antibodies in hydatid disease patients and controls using ELISA test

\begin{tabular}{|l|l|}
\hline Subject groups & Positive results with ELISA (\%) \\
\hline Confirmed cases $(\mathrm{n}=11)$ & $10(90.9)$ \\
\hline Disease controls $(\mathrm{n}=7)$ & $1(14.3)$ \\
\hline Healthy controls $(\mathrm{n}=11)$ & $0(0)$ \\
\hline
\end{tabular}

Table 4. Suspicious cases of amoebic liver abscess and their reaction with ELISA

\begin{tabular}{|l|l|l|}
\hline & Patients with disease & Patients without disease \\
\hline ELISA test positive & 2 & 1 \\
\hline ELISA test negative & 0 & 14 \\
\hline
\end{tabular}

Table 5. Sensitivity and specificity of ELISA test for diagnosis of ALA

\begin{tabular}{|l|l|l|l|l|l|}
\hline Test & Sensitivity & Specificity & PPV & NPV & Diagnostic Efficiency \\
\hline ELISA & $100 \%$ & $93.3 \%$ & 66.7 & 100 & 94.1 \\
\hline
\end{tabular}

Table 6. Detection of anti-amoebic antibodies in amoebic liver abscess patients and control

\begin{tabular}{|l|c|}
\hline \multicolumn{1}{|c|}{ Subject groups } & Positive results with ELISA (\%) \\
\hline Confirmed cases $(\mathrm{n}=11)$ & $10(90.9)$ \\
\hline Disease controls $(\mathrm{n}=7)$ & $1(14.3)$ \\
\hline Healthy controls $(\mathrm{n}=11)$ & $0(0)$ \\
\hline
\end{tabular}



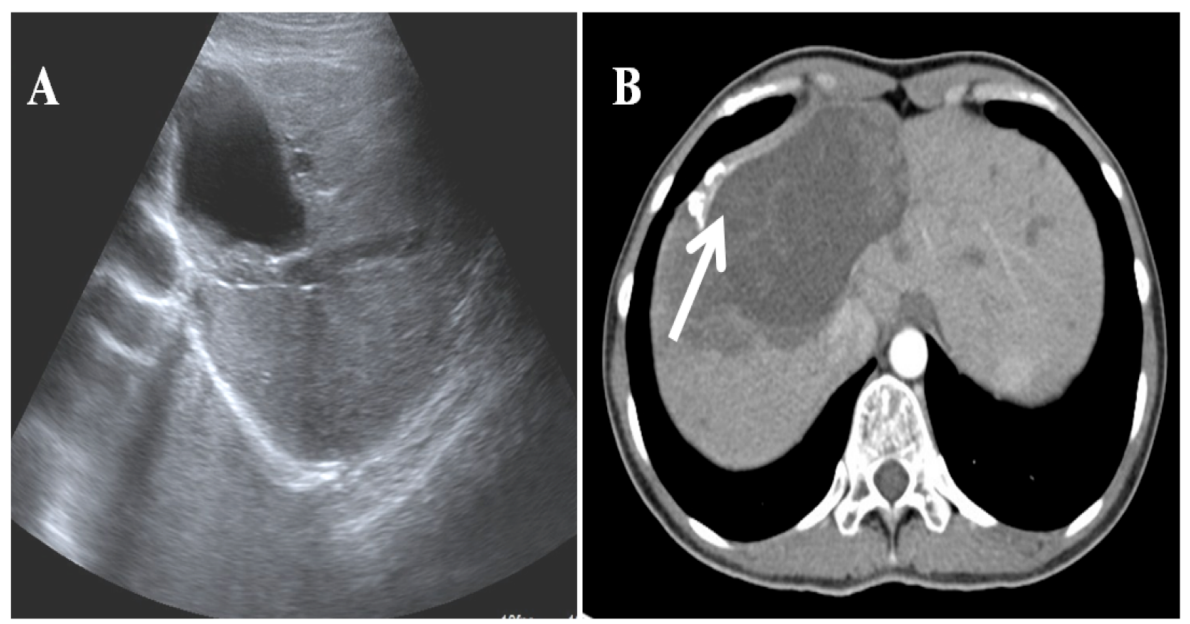

Figure 1: Imaging of the liver:

(A) Ultrasonography of the liver showing cystic lesion

(B) Post-contrast CT for the liver showing right lobe cystic lesion with mural calcification (white arrow).
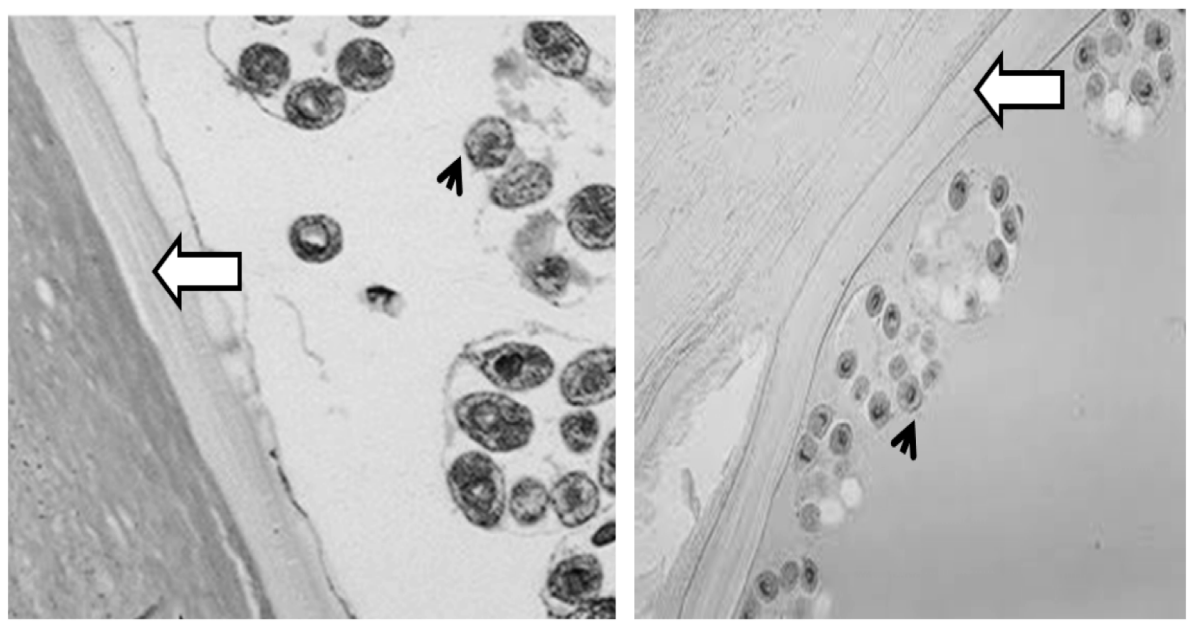

Figure 2 : Histopathological feature of Cystic Echinococcosis. Open arrow refers to cyst wall and arrow heads pointed to protoscolices.

Vol. 43, No. 1 \& 2 Jan. \& April, 2014 


\section{DISCUSSION}

Diagnosis of liver CE is usually accidental, associated with an abdominal sonography performed for other clinical reasons. Presence of symptoms suggestive of $C E$ in a person, who is in contact with dogs, supports the suspicion of the disease. Radiological imaging, especially abdominal ultrasonography, is considered the gold standard for detecting the number, site, size and vitality of the hydatid cyst (8) and it is also important to determine the treatment strategy. But, ultrasonography is not always able to differentiate hydatid cysts from other spaceoccupying lesions, as tumors or abscesses, so additional techniques are required to confirm the radiological diagnosis. Immunodiagnosis is an important technique to validate the imaging diagnosis and can be used for treatment follow up even if there is undetectable immune response (9). The gold standard laboratory tests for human echinococcosis are currently based on standard ELISA or immunoblot using E. granulosus hydatid cyst fluid-derived antigen B (10).

In this study, plates coated with Echinococcus cyst wall antigen for diagnosis of liver CE were used. Although the test was qualitative, it was able to detect CE in $90.9 \%$ of the cases. The obtained sensitivity is nearly similar to those obtained from the most commonly diagnostic tests used for diagnosis of CE based on hydatid fluid antigens, which have relatively high sensitivity for hepatic cysts ranging from $85 \%$ to $95 \%$ (11). More recent, the recombinant form of $\mathrm{AgB}$, a protein derived from cyst fluid, has a sensitivity of $97.1 \%$, but cross reaction with other cestodes were recorded (12) resulting in specificity of $62.7 \%$, which is very low compared to our study (95\%).

Recently, ELISA is being preferred to IHA because of its easy use, low cost and comparable sensitivity. In addition, the tested ELISA kit is simple and no special training was required. Therefore, it has practical value for support of both community mass screening in conjugation with ultrasound, and for hospitalbased diagnostic confirmation of CE.

In our study, only one positive case for hepatic CE was negative by the tested kit. This case had a single small cyst $(2 \mathrm{~cm})$, this data suggested that cyst size could be

MANSOURA MEDICAL JOURNAL 
8 UTILITY OF SEROLOGY IN THE DIAGNOSIS etc...

one of the limitations of this kit diagnosis and limited or even no and only patients cysts bigger cross reactivity.

than $2 \mathrm{~cm}$ are detected using this kit.

One of the important problems encountered was the observation of false positive cases caused by cross-reaction with other helminthic infections. We have recorded only one false positive case due to schistosomiasis mansoni, as Schistosoma probably shares epitopes with cestodes, but we are expecting more cases especially those infected with other cestodes as Taenia species and more parasite-positive controls should be tested for comprehensive evaluation of this kit. CE, especially in the liver, is characterized by the triggering of an intense humoral response with rising titre of antibodies (9). Such response is encouraging for development of novel CE serodiagnostic assays. Although we have plenty of these tests, the used antigens do not provide an appropriate level of sensitivity and specificity. Although the initial evaluation of the new ELISA kit used in this study was promising, since antigens from the cyst wall were used, a single defined recombinant $\mathrm{E}$. granulosus antigen is required urgently to ensure early

Conventional diagnosis of ALA is confirmed by finding the E. histolytica trophozoites in liver pus aspirate obtained via ultrasound guided percutaneous aspiration biopsy, but the parasites are often absent as most of them are located at the margin on the periphery of the abscess (5). Therefore, serodiagnosis is widely adopted for diagnosis of ALA, detecting either amoebic antigens or antibodies from serum samples. However, for antigen detection, available tests showed lower sensitivities ranging from $8.5 \%$ to $50 \%(13,14)$. Hepatic amoebiasis raises a strong humoral immune response especially immunoglobulin $G$ (15) and there are a variety of serological assays used for diagnosis of ALA based on detecting circulating antibodies such as IHA, Latex agglutination, indirect immunofluorescence, counter- immunoelectrophoresis, gel diffusion, complement fixation and ELISA; IHA and ELISA are still the preferred choices (16).

ELISA is commonly used as a routine diagnostic assay in diagnosis of ALA because it can be developed 
in-house use based on different amoebic antigen preparations such as crude soluble antigen (CSA), excretory-secretory antigens, plasma membrane antigens, purified antigenic proteins or recombinant proteins. The CSA-based ELISA technique has been used in routine diagnosis of ALA with reported $100 \%$ sensitivity and more than $90 \%$ specificity (17). In our study, testing a novel ELISA kit utilizing crude amoebic antigens showed $100 \%$ sensitivity, this result is in agreement with the previously recorded results using CSA and better than those recorded when using recombinant proteins $(18,19)$. However, cross-reactivity was reported with serum from patient complaining of Giardia infection with $93.3 \%$ specificity. False positive diagnosis of ALA reported in our study could be attributed to variability in the antigen preparations since this antigens prepared from amoeba maintained in the culture and the proteins used for diagnosis may vary from patch to patch. Although several proteins have been evaluated by ELISA technique for diagnosis of ALA, they were less sensitive and immunoreactivity have been reported with amoebic dysentery and even asymptomat- ic cases (20). In the current study, this new test showed promising diagnostic validity.

In conclusion, in this work, we have detected that ELISA could be useful for diagnosis of hepatic CE and ALA; however a future study with more patients is needed to assess the validity of kits used in this study.

\section{REFERENCES}

1. Vachha, B., Sun, M.R.M., Siewert, B., and Eisenberg, R.L. (2011) : Cystic lesions of the liver. Am J Roentgenol 196, W355-W

2. Macedo, F.I. (2013) : Current management of noninfectious hepatic cystic lesions: A review of the literature. World J Hepatol 5, 462469.

3. Sadjjadi, S.M. (2006) : Present situation of echinococcosis in the Middle East and Arabic North Africa. Parasitol Int 55, S197-S202.

4. Reid Lombardo, K., Khan, S., and Sclabas, G. (2010) : 
Hepatic cysts and liver abscess. Surg Clin North Am 90, 679-697.

5. Stanley Jr, S.L. (2003) : Amoebiasis. Lancet 361, 10251034.

6. Salles, J.M., Moraes, L.A., and Salles, M.C. (2003) : Hepatic amebiasis. The Brazilian journal of infectious diseases : an official publication of the Brazilian Society of Infectious Diseases 7, 96-110.

7. Wells, C.D., and Arguedas, M. (2004) : Amebic liver abscess. South Med J 97, 673-682.

8. Richter, J., Hatz, C., and Häussinger, D. (2003) : Ultrasound in tropical and parasitic diseases. Lancet 362, 900-902.

9. Zhang, W., and McManus, D.P. (2006) : Recent advances in the immunology and diagnosis of echinococcosis. FEMS Immunol Med Microbiol 47, 24-41.
10. Zheng, G.Y., Zhao, R.L., and Feng, X.H. (1986) : Dotimmunobinding assay in the serodiagnosis of human hydatid disease. Am J Trop Med Hyg 35, 812814.

11. Siracusano, A., and Bruschi, F. (2006) : Cystic echinococcosis: Progress and limits in epidemiology and immunodiagnosis. Parassitologia 48, 65-66.

12. Tamarozzi, F., Sako, Y., Ito, A., Piccoli, L., Grisolìa, A., Itoh, S., Gatti, S., Meroni, V., Genco, F., and Brunetti, E. (2013) : Recombinant AgB8/1 ELISA test vs. commercially available $\lg G$ ELISA test in the diagnosis of cystic echinococcosis. Parasite Immunol 35, 433440.

13. Parija, S.C., and Khairnar, K. (2007) : Detection of excretory Entamoeba histolytica DNA in the urine, and detection of $E$. histolytica DNA and lectin antigen in the liver abscess pus for 
the diagnosis of amoebic liver abscess. BMC Microbiol 7 .

14. Zeehaida, M., Wan Nor Amilah, W.A.W., Amry, A.R., Hassan, S., Sarimah, A., and Rahmah, N. (2008) : A study on the usefulness of techlab Entamoeba histolytica II antigen detection ELISA in the diagnosis of amoebic liver abscess (ALA) at Hospital Universiti Sains Malaysia (HUSM), Kelantan, Malaysia. Tropical Biomed 25, 209-216.

15. Valenzuela, O., Ramos, F., Morán, P., González, E., Valadez, A., Gómez, A., Melendro, E.I., Ramiro, M., Muñoz, O., and Ximénez, C. (2001) : Persistence of secretory antiamoebic antibodies in patients with past invasive intestinal or hepatic amoebiasis. Parasitol Res 87, 849-852.

16. Fotedar, R., Stark, D., Beebe, N., Marriott, D., Ellis, J., and Harkness, J. (2007) :
Laboratory diagnostic techniques for Entamoeba species. Clin Microbiol Rev 20, 511-532.

17. Tachibana, H., Cheng, X.J., Masuda, G., Horiki, N., and Takeuchi, T. (2004) : Evaluation of Recombinant Fragments of Entamoeba histolytica Gal/GalNAc Lectin Intermediate Subunit for Serodiagnosis of Amebiasis. J Clin Microbiol 42, 1069-1074.

18. Shenai, B.R., Komalam, B.L., Arvind, A.S., Krishnaswamy, P.R., and Subba Rao, P.V. (1996) : Recombinant antigen-based avidin-biotin microtiter enzyme-linked immunosorbent assay for serodiagnosis of invasive amebiasis. J Clin Microbiol 34, 828-833.

19. Haghighi, A., and Rezaeian, M. (2005) : Detection of serum antibody to Entameba histolytica in various population samples of amebic infection using an enzyme-

MANSOURA MEDICAL JOURNAL 
12 UTILITY OF SEROLOGY IN THE DIAGNOSIS etc...

linked immunosorbent as- of amoebiasis using a resay. Parasitol Res 97, combinant protein fragment 209-212. of the $29 \mathrm{kDa}$ surface antigen of Entamoeba histolyti-

20. Lee, J., Park, S.J., and Yong, ca. Int J Parasitol 30, T.S. (2000) : Serodiagnosis 1487-1491.

Vol. 43, No. 1 \& 2 Jan. \& April, 2014 